\title{
Ayahuasca : liane de l'âme, chamanes et soumission chimique
}

\section{Ayahuasca : spirit liana, shamans and chemical submission}

Gilbert PÉPIN, Gaëlle DUFFORT

Laboratoire d'Expertises TOXLAB, 7, rue Jacques Cartier - 75018 PARIS Tél : 0158592800 - Fax : 0158592801 - E-mail : toxlab@wanadoo.fr

(Reçu le 30 janvier 2004 ; accepté le 10 février 2004)

\section{$R \mathbf{E} S \boldsymbol{U} M E^{\prime}$}

Les plantes hallucinogènes sont utilisées dans les sociétés primitives depuis des siècles, mais jusqu'à maintenant leur usage se limitait à quelques individus chamanes, médecinssorciers, etc... lors de rites religieux. Actuellement un regain d'intérêt est constaté pour les hallucinogènes naturels en rapport avec un mouvement de "retour à la nature " car la diffusion de ces substances est depuis peu largement facilitée et accélérée par l'intermédiaire d'internet. Parmi celles-ci l'Ayahuasca est la plus connue.

Il s'agit d'un mélange de plusieurs plantes du nord-ouest de l'Amérique du Sud. Pour sa préparation, deux espèces de Banisteriopsis, liane géante, sont particulièrement importantes et sources de $\beta$-carbolines (harmaline, harmine, tétrahydroharmine et harmol qui sont des hallucinogènes naturels). Généralement une autre plante est ajoutée afin de modifier l'intensité, la durée et les effets des $\beta$-carbolines. Cette autre plante varie selon la région géographique et renferme plutôt des principes actifs riches en dérivés de la tryptamine, telle que la Psychotria Viridis qui contient principalement de la diméthyltryptamine (DMT) aux propriétés hallucinogènes. L'utilisation par les indiens d'Amérique du Sud d'une autre plante, la Virola, contenant des dérivés de la DMT sera également développée ici.

La mise en évidence directe des principes actifs hallucino-

\section{SUMMARY}

Hallucinogenic plants are used in the primitive societies for centuries, but so far they were only used by some shamans, healers, etc. during religious rites. Nowadays a renewal of interest is noticed for natural hallucinogens in connection with a return-to-nature movement because the spreading of these substances has been recently more easier and increased through internet. Among these hallucinogens, Ayahuasca is the most well-known.

Ayahuasca is a mixture of several plants from the northwest of South America. For its preparation, two sorts of Banisteriopsis, giant liana, are particularly important and sources of $\beta$-carboline alkaloids (harmaline, harmine and tétrahydroharmine, harmol which are natural hallucinogens). Generally, another plant is added to modify intensity, duration and effects of $\beta$-carbolines. This natural additive varies according to the region and contains predominantly active principles, rich in tryptamine's metabolites, such as Psychotria viridis which contains mainly dimethyltryptamine (DMT) with hallucinogenic properties. The use by the Indians of South America of another plant, Virola, containing by-products of the DMT will be also developed here.

The direct analysis of the hallucinogenic active principles can be realized by a half and half dilution in ethanol, then detection and quantification can be realized by gas chroma- 
gènes peut être réalisée par dilution au demi de la solution à analyser dans de l'éthanol, puis la détection et la quantification peuvent être réalisées au moyen de la chromatographie en phase gazeuse couplée à un spectromètre de masse. La détection et la quantification dans le plasma humain des carbolines peut s'effectuer par chromatographie en phase liquide haute performance après précipitation des protéines plasmatiques et une détection par fluorescence. La DMT peut être dosée dans le plasma par chromatographie en phase gazeuse avec une détection azote-phosophore après extraction liquide-liquide par le chlorobutane à pH basique, ou par chromatographie en phase gazeuse couplée à la spectrométrie de masse (CPG/SM).

L'association de ces substances provoque une profonde altération de l'état de conscience. Il y a peu d'altération de la perception réelle de l'environnement hormis quelques améliorations des fonctions sensorielles générales. Après une période de vertige, de nausées, de nervosité, de transpiration et de manifestations digestives parfois violentes, l'ingestion d'ayahuasca entraîne des hallucinations sensorielles diverses suivies d'une période de lassitude ; il s'installe ensuite parfois un sommeil fiévreux, entrecoupé de rêves. Les risques liés à l'utilisation d'un hallucinogène aussi puissant que l'ayahuasca, outre l'interaction avec les antidépresseurs sérotoninergiques, sont essentiellement d'ordre psychologique : elle peut être un important facteur de déstructuration psychologique. Elle autorise également une forme de soumission chimique des adeptes par les responsables des sectes. C'est pourquoi le ministère de la santé publique français à classé la DMT et ses dérivés comme produits stupéfiants.

\section{MOTS-CLÉS}

Ayahuasca, hallucinogènes, diméthyltryptamine, béta-carbolines, chamanes, soumission chimique.

\section{Introduction}

Les plantes hallucinogènes sont utilisées par les sociétés primitives depuis l'époque protohistorique, mais jusqu'à maintenant leur usage se limitait à un cercle restreint d'indiens d'Amérique du Sud en particulier dans le cadre de pratiques mystico-religieuses, lors de circonstances exceptionnelles telles que l'initiation des jeunes adolescents à la fin de la puberté, ou pour ses vertus thérapeutiques. Depuis quelques années, de nouveaux hallucinogènes naturels sont apparus sur le marché des drogues récréatives, s'inscrivant dans un mouvement de retour à la nature de plus en plus prisé. Autre vecteur de la propagation de ces substances, leur mise à disposition sur internet n'a fait qu'accélérer cette récente émergence. Parmi ces hallucinogènes naturels, l'ayahuasca ou liane de l'âme, en provenance du bassin de l'Amazonie connaît un nouvel essor en Europe. Malgré son utilisation très ancienne, la question de sa dangerosité se pose car des exemples de soumissions chimiques en groupe organisé ont été rapportés sous l'emprise d'ayahuasca. tography coupled with a mass spectrometer (CG/MS). Detection and quantification of carbolines in human plasma can be done by means of high-performance liquid chromatography (HPLC) with fluorescence detection after blood proteins precipitation. DMT can be determined in plasma by gas chromatography with selective nitrogen - phosphorus or mass spectrometer detection following liquid - liquid extraction with chlorobutane at alkaline $\mathrm{pH}$.

This carbolines and DMT association generate a deep change of the consciousness state. Only a few changes of the perception of the environment are observed, but some improvements of the general sensory functions occur. After a period of dizziness, sickness, nervousness, perspiration and sometimes violent digestive symptoms, the ingestion of ayahuasca provokes different sensory hallucinations followed by a period of tiredness; then sometimes a feverish sleep settles down, interrupted with dreams.

Besides interaction with serotoninergic antidepressants, the risks related to the use of such a powerful hallucinogen as ayahuasca, are essentially psychological: it can led the mind to become destructured. It also authorizes a kind of chemical submission of the followers by sects responsibles. That's why the French ministry of health classified the DMT and its byproducts as narcotics.

\section{KEY-WORDS}

Ayahuasca, hallucinogenics, dimethyltryptamine, beta-carbolines, shamans, chemical submission.

\section{Chimie des hallucinogènes}

A l'exception du Cannabis sativa, aux propriétés sensiblement différentes, les végétaux hallucinogènes doivent leurs propriétés à des substances azotées (structures indoliques, indoalkylamines ou alcaloïdes vrais). Il existe une analogie structurale étroite entre ces substances hallucinogènes et certains neuromédiateurs du cerveau humain (sérotonine, adrénaline, noradrénaline) ; cette parenté de structure chimique explique en partie le pouvoir psychotrope de ces substances hallucinogènes (1).

\section{Ayahuasca, liane de l'âme ou liane des morts}

Parmi les drogues hallucinogènes à alcaloïdes indoliques, une famille de plantes ligneuses tropicales fournit deux espèces hallucinogènes connues sous le nom d'ayahuasca (Equateur, Pérou) ou de caapi (Brésil). Elles sont largement consommées en Amérique du Sud, principalement dans l'ouest du bassin de l'Amazonie, sur le versant de la cordillère des Andes, 
par des tribus isolées du versant pacifique de la Colombie, de l'Equateur et du Pérou. Le terme ayahuasca vient de la langue amérindienne Quechua où aya signifie mort et par extension âme, et huasca signifie corde et par extension liane (2). Ce terme désigne à la fois une plante (Banisteriopsis caapi) mais également la boisson (issue d'une décoction) dont elle est toujours le constituant soit seule, soit additionnée d'autres plantes.

\section{Botanique}

L'ayahuasca (boisson) peut être réalisée à partir de plusieurs espèces de plantes de la famille des Malpighiacées : en particulier Banisteriopsis caapi (Figure 1) et Banisteriopsis inebrians. Ces deux espèces sont des lianes géantes à écorce lisse et brune. La décoction de ces écorces peut être bue telle quelle, mais diverses autres plantes sont souvent ajoutées à cette boisson pour en modifier la nature et l'intensité des effets. Les additifs végétaux peuvent être d'autres Malpighiacées telles que Diplopterys cabrerana, mais les plus couramment rencontrés sont les feuilles de chacruna, un arbuste de la famille des Rubiacées : Psychotria viridis, croissant dans les mêmes régions tropicales que Banisteriopsis et très fréquemment associée à $B$. caapi ; mais d'autres plantes psychotropes y sont parfois mêlées telle que Brugmansia suaveolens (plante arborescente très proche de la Datura) (3), des feuilles de B. rusbyana ou Oco-Yagé.

Les feuilles de Banisteriopsis caapi sont vert foncé, lancéolées, longues de $18 \mathrm{~cm}$ et larges de 5 à $8 \mathrm{~cm}$. L'inflorescence est constituée de nombreuses fleurs de petite taille et de couleur allant du rose pâle au rose foncé. Le fruit est un samare de 3 à $5 \mathrm{~cm}$. Des feuilles ovales et plus épaisses distinguent $B$. inebrians de $B$. caapi.

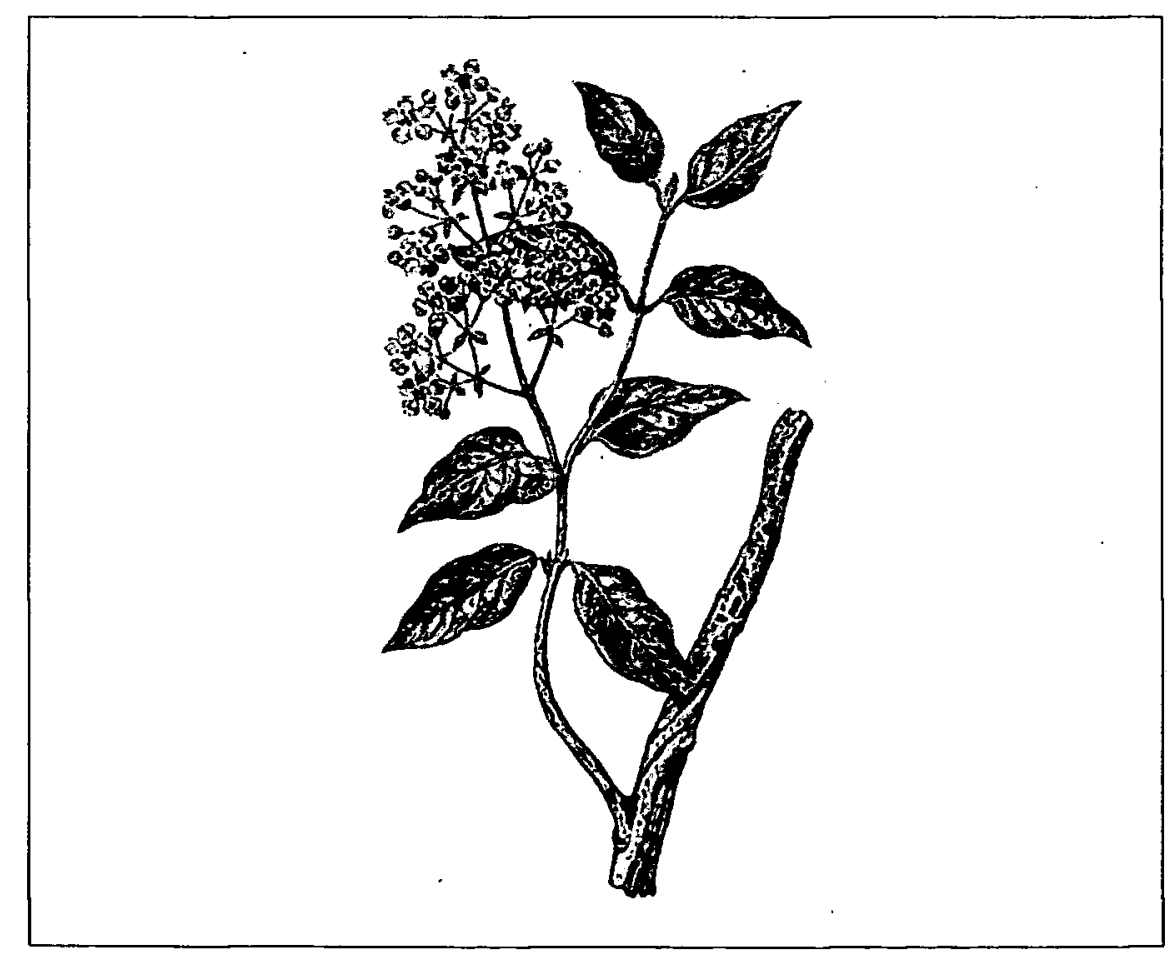

Figure 1 : Dessin de Banisteriopsis caapi par C.B. Robinson et Small (3).
Divers procédés sont employés pour préparer cette décoction hallucinogène, le plus souvent l'écorce de morceaux de tiges fraîchement coupés est grattée ; dans l'ouest de l'Amérique du Sud, l'écorce est bouillie pendant plusieurs heures, l'épais liquide très amer est alors absorbé à petites doses. Ailleurs, l'écorce est grattée puis pulvérisée et pétrie dans l'eau froide, dans ce cas, des doses plus élevées peuvent être absorbées car elle est moins concentrée.

\section{Principes actifs, relation structure-activité}

Si l'harmaline et l'harmine ont été isolées il y a plus d'un siècle (en 1841), dans les graines de Peganum harmala (ou Rue de Syrie), une plante de la famille des Zygophyllacées traditionnellement utilisée au MoyenOrient (Syrie) pour ses propriétés médicinales, la structure chimique de l'harmaline a été établie en 1919 et sa synthèse chimique fut réalisée pour la première fois en 1927 par Richard Manske. Puis la connaissance précise de la composition chimique de l'ayahuasca est apparue en 1957 avec les travaux de Hochstein et Paradies ; ces découvertes ont été quantitativement confirmées par L. Rivier et J.E. Lindgren en 1972 (4). Les alcaloïdes totaux de Banisteriopsis caapi représentent en moyenne $0.5 \%$ et sont constitués de $\beta$-carbolines : harmine, tétrahydroharmine, harmaline, harmol, etc..., qui sont des alcaloïdes dérivés de la tryptamine aux propriétés hallucinogènes (Figure 2).

La plante Psychotria viridis contient des alcaloïdes de type dimétyltryptamine ou DMT (Figure 3). La DMT est un agent hallucinogène agoniste des récepteurs sérotoninergiques 5-HT (analogue structurel du LSD). Plusieurs chercheurs ont d'abord avancé que les propriétés de la décoction d'ayahuasca étaient uniquement dues à la combinaison $\beta$-carbolines - DMT. En effet, la DMT est active par voie intramusculaire, mais par voie orale elle n'a pas d'effet pharmacologique car elle subit une inactivation par une enzyme largement répandue dans l'organisme, la monoamine oxydase. Les $\beta$-carbolines ayant des propriétés d'inhibition des monoamines oxydases (IMAO), l'association $\beta$-carbolines - DMT permettrait donc à la DMT d'exercer son action y compris par voie orale et de la potentialiser. Depuis, un nouveau schéma de protection de la DMT a été proposé par McKenna et Towers (1985) faisant référence à différents inhibiteurs d'oxydases présents dans cette plante ; cependant cette hypothèse n'a pas été vérifiée (5). L'action inhibitrice de la monoamine oxydase des $\beta$-carbolines s'est avérée modifier également un paramètre majeur de la pharmacocinétique de la DMT ingérée dans la décoction ayahuasca : son temps de concentration plasmatique maximal passe à $1 \mathrm{~h} 30$ en moyenne alors qu'il est de 10 à 15 minutes par voie intramusculaire et de 2 minutes par voie intraveineuse ! (2) 
Substances hallucinogènes naturelles puissantes, les tryptamines ont également été identifiées dans l'écorce d'une autre plante utilisée par les indiens d'Amazonie, du genre Virola.<smiles>COc1ccc2c(c1)[nH]c1c(C)nccc12</smiles>

Harmine<smiles>COc1ccc2c3c([nH]c2c1)C(C)NCC3</smiles>

Tétrahydroharmine<smiles>COc1ccc2c3c([nH]c2c1)C(C)=NCC3</smiles>

Harmaline<smiles>Cc1nccc2c1[nH]c1cc(O)ccc12</smiles>

Harmol

Figure 2 : Formules chimiques des b-carbolines contenues dans Banisteriopsis caapi : harmine, tétrahydroharmine, harmaline, harmol.<smiles>CN(C)CCc1c[nH]c2ccccc12</smiles>

Diméthyltryptamine (DMT)

Figure 3 : Formule chimique de la Diméthyltryptamine $(D M T)$, composant de Psychotria viridis et Virola.

\section{Virola ou Semence du soleil}

Bien qu'une soixantaine d'espèces de Virola existent dans les régions tropicales de l'Amérique centrale et du Sud, c'est seulement dans l'ouest de l'Amazonie, autour du bassin de l'Orénoque que le genre Virola (encore appelé epená, yakee, paricá, nyakwana) est à l'origine d'une utilisation en tant qu'hallucinogène sacré. La poudre à priser hallucinogène qui en est tirée permettrait à ceux qui l'absorbent (les plus souvent des chamanes) d'entrer en contact avec le monde des esprits.

\section{Botanique}

De la famille des Myristicacées, apparenté aux Muscadiers, le genre Virola regroupe de petits arbres élancés de 7,5 à 20 mètres de haut. Le tronc cylindrique peut rappeler les lianes et possède une écorce caractéristique très lisse, brun tacheté de gris. Les feuilles, qui une fois sèches dégagent une odeur de thé, sont ovales, lancéolées, longues de 9 à $33 \mathrm{~cm}$ et larges de 4 à $11 \mathrm{~cm}$ (Figure 4 et 5). Les inflorescences mâles très fournies sont velues, brunes ou jaune doré, plus courtes que les feuilles. Les fleurs, toutes petites, en groupe de 2 à 10 sont très odorantes. Le fruit est presque rond, mesurant de 1 à $2 \mathrm{~cm}$. La graine est à moitié recouverte par une arille rouge orangé. L'aubier de la plupart des espèces de Virola produit une abondante résine rouge après écorçage et séchage au feu. Quelques tribus du cour supérieur de l'Orénoque réduise la préparation en une poudre à priser ou des petites pastilles aux propriétés hallucinogènes. Les espèces utilisées sont $V$. calophyl$l a$, V. elongata, et $V$. theiodora, cette dernière étant la plus fréquemment employée.

\section{Principes actifs, relation structure-activité}

Cet hallucinogène a été mentionné pour la première fois au début du siècle (1909) par un ethnologue allemand, Koch-Grünberg qui en signale l'usage chez les Indiens Yekwana du haut Orénoque, ce n'est qu'en 1938 que le botaniste Brésilien Ducke fait le rapprochement entre les Virola et la poudre à priser.(6)

L'analyse chimique des diverses espèces de Virola a révélé une demi-douzaine d'alcaloïdes indoliques apparentés appartenant aux dérivés de tryptamines à système tétrahydro $\beta$-carbolines. Des travaux de Holmstedt et Lindgren (1967) ont permis d'identifier précisément la nature de ces dérivés de la tryptamine dans la Virola (7).

Les principaux composants de ces diverses poudres à priser sont : la N,N-diméthyltryptamine (DMT) (Figure 3), la 5-méthoxy-N,N-diméthyltryptamine (5-MeODMT) et la N-méthyltryptamine (MMT)..., (Figure 6). La drogue en contient parfois des quantités considérables (jusqu'à $11 \%$ dans certains échantillons). 


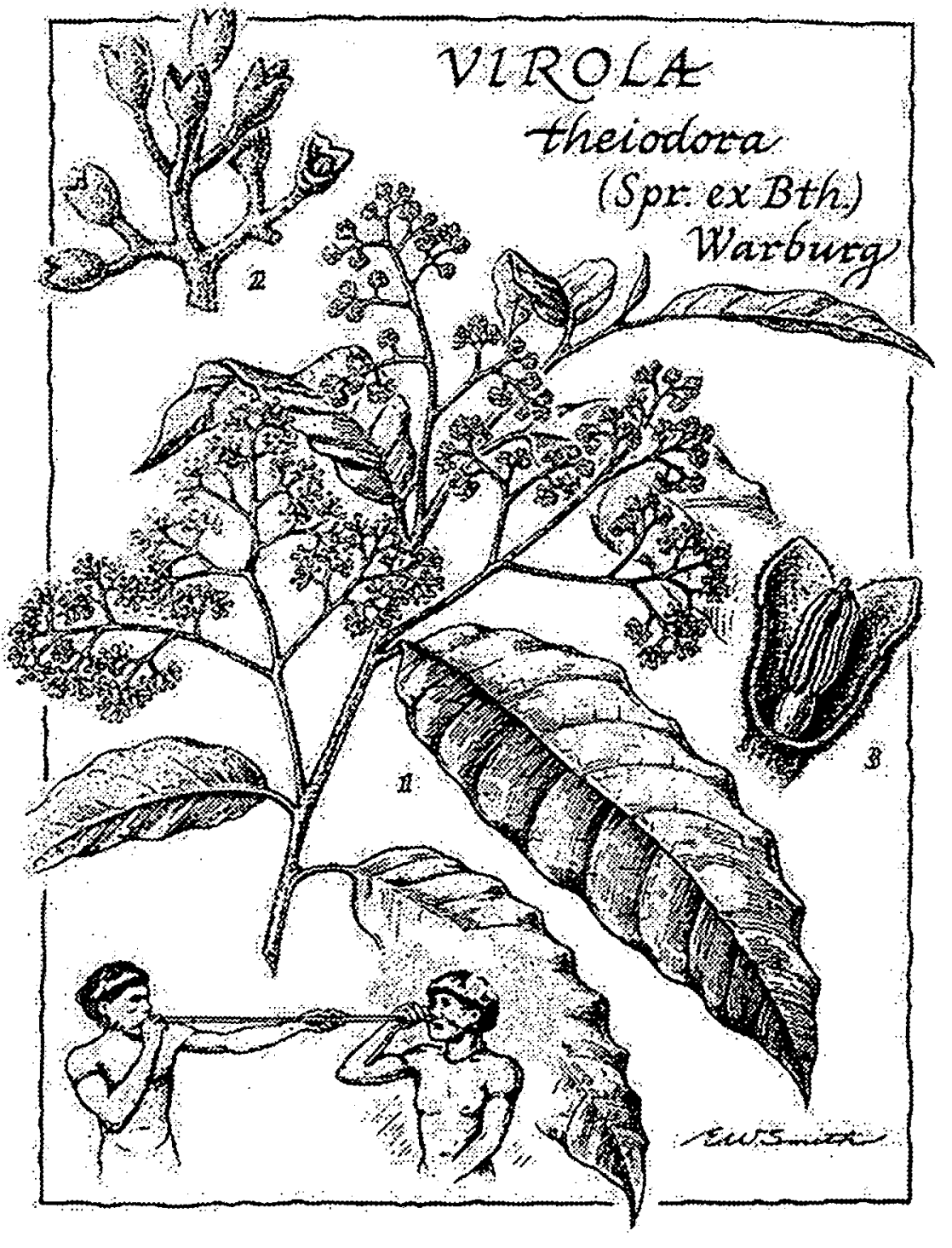

Figure 4: Dessin de Virola theiodora par E.W. Smith (6).

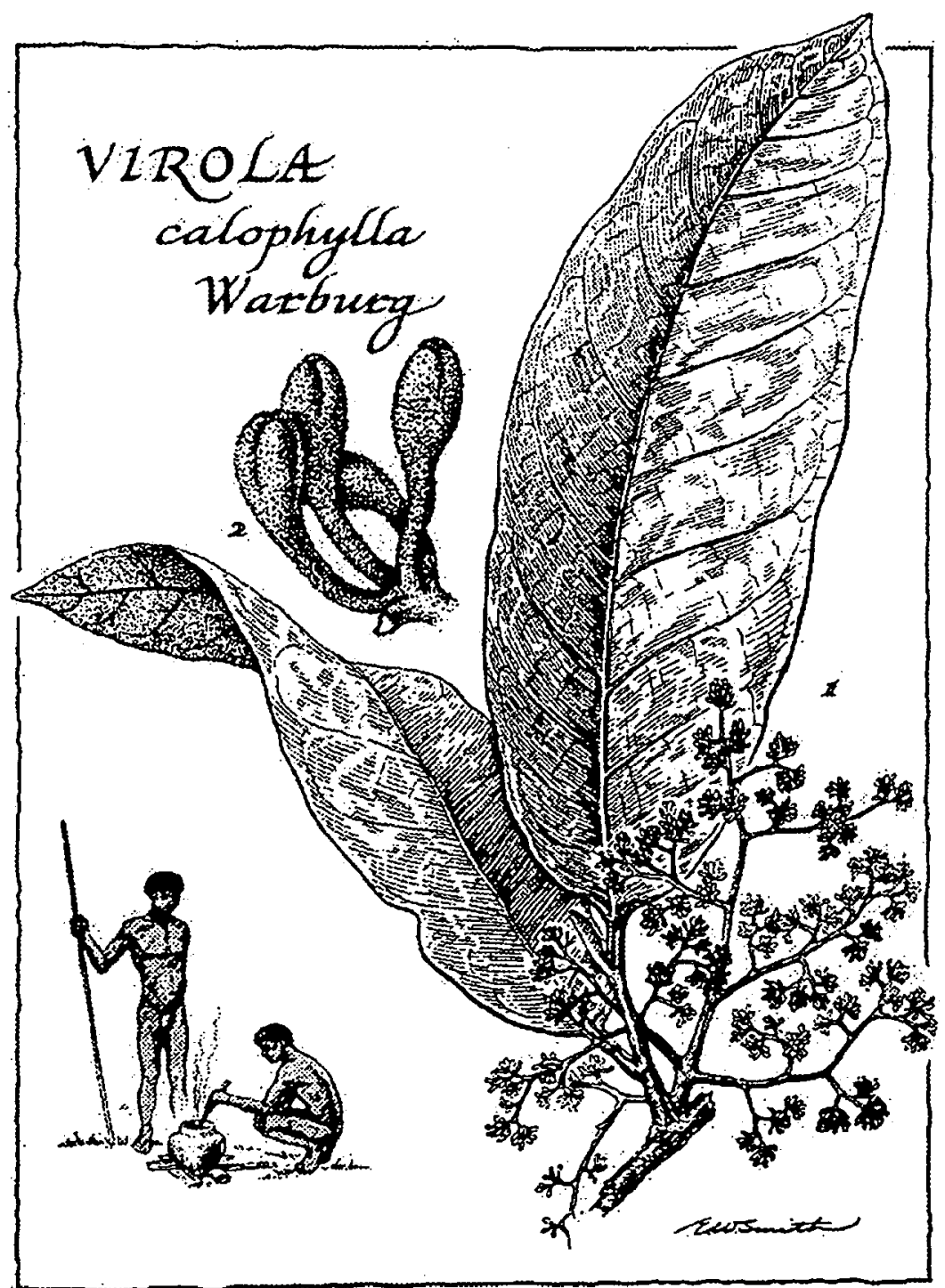

Figure 5 : Dessin de Virola calophylla Warburg par E.W. Smith (6).

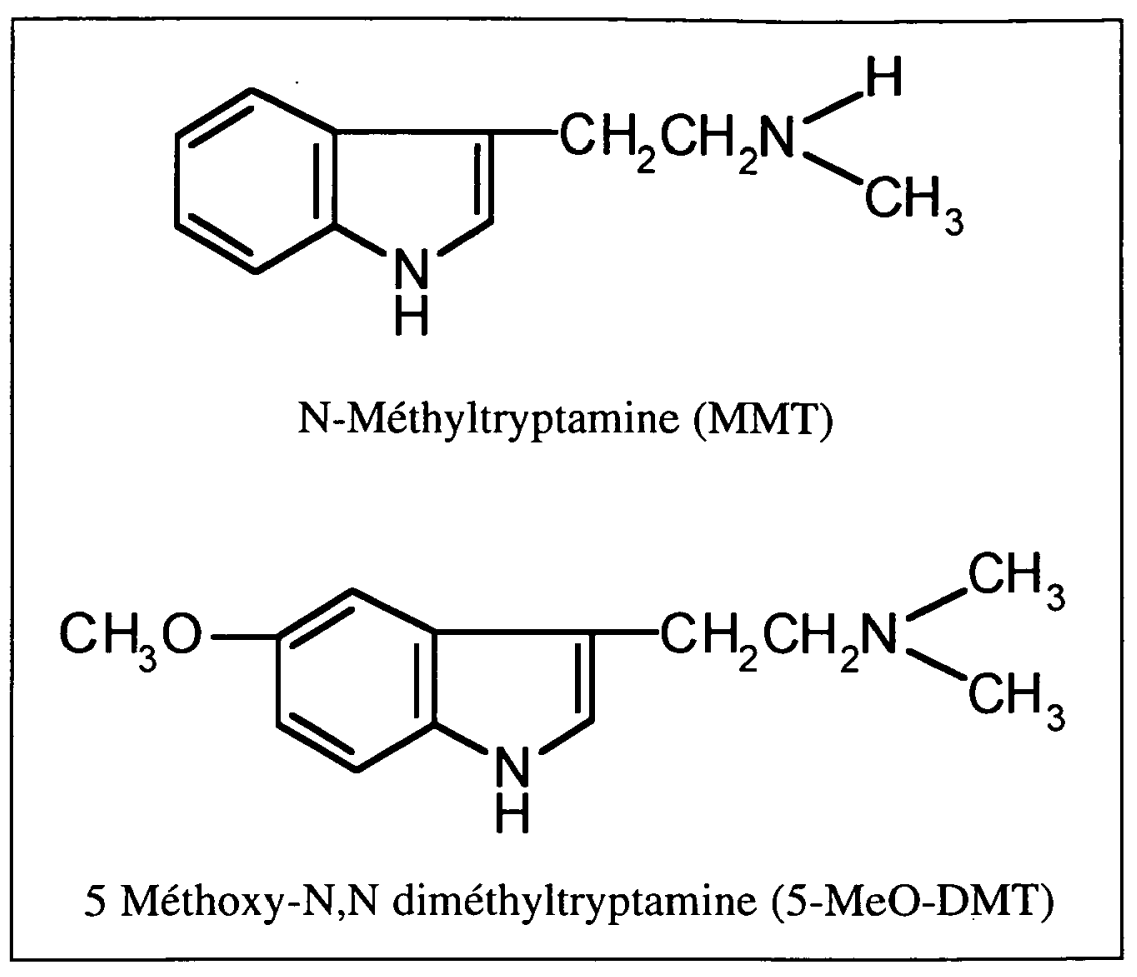

Figure 6 : Formule chimique des tryptamines identifiées dans les Virola.

\section{Détection de la DMT et des b-carbolines dans les milieux biologiques}

Une méthode de détection et de dosage dans le sérum des tryptamines et des $\beta$-carbolines a été décrite par Callaway en 1996 (8). En 2002, Yritia et coll. ont proposé la procédure de dosage dans le sérum suivante (9): la DMT est dosée dans le plasma humain par chromatographie en phase gazeuse couplée à un détecteur azotephosphore (CPG/NPD) (des méthodes utilisant un détecteur de masse, CPG/SM, ont également été décrites) (4), les échantillons sont injectés en mode splitless après ajout d'un étalon interne et extraction liquide-liquide par le n-pentane à pH basique. Les limites de détection et de quantification de la DMT obtenues avec cette méthode sont respectivement 0.5 et $1.6 \mathrm{ng} / \mathrm{ml}$.

Les $\beta$-carbolines peuvent être dosées dans le plasma humain par chromatographie en phase liquide couplée à un détecteur à fluorescence (CLHP/FD) après ajout d'un étalon interne et extraction solide-liquide sur cartouche (acétonitrile/eau et triéthylamine).

Les limites de détection obtenues avec cette méthode sont de $0.1 \mathrm{ng} / \mathrm{ml}$ pour l'harmine et $0.3 \mathrm{ng} / \mathrm{ml}$ pour l'harmaline, l'harmol et l'harmalol ; les limites de quantification sont de $0.5 \mathrm{ng} / \mathrm{ml}$ pour l'harmine et 0.3 $\mathrm{ng} / \mathrm{ml}$ pour l'harmaline, l'harmol et l'harmalol.

La méthode de dosage de Callaway appliquée à 15 volontaires ayant consommé de l'ayahuasca $(2 \mathrm{ml} / \mathrm{kg}$ contenant harmine $1,7 \mathrm{mg} / \mathrm{ml}$, harmaline $0,2 \mathrm{mg} / \mathrm{ml}$, tétrahydroharmine $1,1 \mathrm{mg} / \mathrm{ml}$ et DMT $0,25 \mathrm{mg} / \mathrm{ml}$ ) au cours d'un rite religieux, a permis de mesurer les concentrations sériques moyennes suivantes : harmine $114,6 \mathrm{ng} / \mathrm{ml}$, harmaline $6,2 \mathrm{ng} / \mathrm{ml}$, tétrahydroharmine $90,8 \mathrm{ng} / \mathrm{ml}$ et DMT $15,8 \mathrm{ng} / \mathrm{ml}(8)$. 
Notons que depuis le milieu des années 60 , plus d'une demi-douzaine d'équipes de chercheurs en psychiatrie biologique ont mis en évidence des taux physiologiques de diméthyltryptamine (DMT) dans différents milieux biologiques humains tels que le sang (10), le plasma (11), l'urine (12) et le liquide céphalo-rachidien (13). Ceci fait de la diméthyltryptamine le premier endo-alcaloïde potentiellement psychoactif mis en évidence dans l'organisme humain. De même, des $\beta$-carbolines endogènes ont été détectées dans le système nerveux central de mammifères, en particulier de l'harmine a été récemment mise en évidence dans le sang humain (14) et la pinoline (6-MeOTHBC) dans la glande pinéale (15).

\section{Identification de principes actif dans des produits de saisie}

Lors d'une saisie au siège d'une secte à Paris de plusieurs bouteilles et jerrycans contenant un liquide laiteux de couleur marron-orangé, les principes actifs de l'ayahuasca ont été mis en évidence.

Après dilution au demi de la solution à étudier dans de l'éthanol, les échantillons ont été analysés en CPG/SM. Les analyses ont montré la présence de DMT, harmine et harmaline dans tous les récipients et dans des proportions communément décrites par d'autres auteurs dans des travaux concernant l'ayahuasca (16).

Le breuvage contient habituellement entre 300 et 800 $\mathrm{mg}$ de carbolines par dose et entre 40 et $80 \mathrm{mg}$ de DMT. F. Bois-Mariage a établi, en 2002, une synthèse des recherches comportant des analyses quantitatives par CPG/SM ou CLHP, d'ayahuasca consommé au sein de tribus indiennes ou d'églises syncrétiques en Amérique du Sud, établissant ainsi les concentrations et doses moyennes par prise d'ayahuasca, des principaux alcaloïdes rencontrés. (Tableau I) (2).

Tableau I : D'après F. Bois-Mariage, Analyses quantitatives d'ayahuasca : concentrations et doses par prise moyennes des principaux alcaloïdes.

\begin{tabular}{|l|c|l|l|l|l|}
\hline Contexte & $\mathrm{N}$ & $\begin{array}{l}\text { Harmine } \\
(\mathrm{mg} / \mathrm{ml}) \\
\text { Dose/prise }\end{array}$ & $\begin{array}{l}\text { THH } \\
\text { (mg/ml) } \\
\text { Dose/prise }\end{array}$ & $\begin{array}{l}\text { DMT } \\
(\mathrm{mg} / \mathrm{ml}) \\
\text { Dose/prise }\end{array}$ & $\begin{array}{l}\text { Techniques } \\
\text { analytiques }\end{array}$ \\
\hline $\begin{array}{l}\text { Indiens Kulina et } \\
\text { Sharanawa, Rio } \\
\text { Purus, Pérou }\end{array}$ & 6 & $\begin{array}{l}0.14 \pm 0.06 \\
30 \mathrm{mg}\end{array}$ & $\begin{array}{l}0.05 \pm 0.03 \\
10 \mathrm{mg}\end{array}$ & $\begin{array}{l}0.12 \pm 0.04 \\
25 \mathrm{mg}\end{array}$ & CPG-SM \\
\hline $\begin{array}{l}\text { Guérisseurs métis de } \\
\text { Pulcallpa, Pérou }\end{array}$ & 5 & $\begin{array}{l}4.67 \pm 0.2 \\
280 \mathrm{mg}\end{array}$ & $\begin{array}{l}1.6 \pm 0.08 \\
96 \mathrm{mg}\end{array}$ & $\begin{array}{l}0.6 \pm 0.06 \\
36 \mathrm{mg}\end{array}$ & $\begin{array}{l}\text { CLHP } \\
\text { Quantitative }\end{array}$ \\
\hline $\begin{array}{l}\text { Santo Daime } \\
\text { (Amérique du Sud) }\end{array}$ & 1 & $\begin{array}{l}1.49 \\
112 \mathrm{mg}\end{array}$ & $\begin{array}{l}1.39 \\
104 \mathrm{mg}\end{array}$ & $\begin{array}{l}0.53 \\
40 \mathrm{mg}\end{array}$ & CPG-SM \\
\hline $\begin{array}{l}\text { Santo Daime, Céu } \\
\text { do Mar, Rio de }\end{array}$ & 4 & $\begin{array}{l}0.74 \\
56 \mathrm{mg}\end{array}$ & $\begin{array}{l}0.58 \\
44 \mathrm{mg}\end{array}$ & $\begin{array}{l}0.55 \\
41 \mathrm{mg}\end{array}$ & $\begin{array}{l}\text { CLHP } \\
\text { Quantitative }\end{array}$ \\
\hline $\begin{array}{l}\text { Uniao do Vegetal, } \\
\text { Nucleo Caupuri, }\end{array}$ & 1 & $\begin{array}{l}1.70 \\
252 \mathrm{mg}\end{array}$ & $\begin{array}{l}1.07 \\
159 \mathrm{mg}\end{array}$ & $\begin{array}{l}0.24 \\
35,5 \mathrm{mg}\end{array}$ & $\begin{array}{l}\text { CLHP } \\
\text { Quantitative }\end{array}$ \\
\hline
\end{tabular}

$\mathrm{N}=$ nombre d'échantillons

\section{Mode de consommation et effets sur l'homme}

\section{Ayahuasca}

L'usage de ce type de boissons sacrées est répandu depuis plus de 5000 ans dans de nombreuses tribus indigènes sud-américaines où elles sont connues sous divers noms (plus de 80 noms différents tels que Yage en Colombie, Caapi au Brésil, yapé, yakee, natem, natema, parica, epéna, nyakwana...). Ce breuvage qui sert à la fois à la divination, à la sorcellerie et à la thérapeutique est profondément enraciné dans la mythologie et la philosophie indigène.

Ce sont les chamanes (sorciers-guérisseurs) qui, représentant l'autorité spirituelle d'une communauté, guérissent les âmes et les corps sous l'influence de cette drogue et en deviennent les spécialistes après un apprentissage long et difficile. Celle-ci leur permettrait au cours de cérémonies curatives de diagnostiquer des maladies, éloigner les désastres imminents, deviner les desseins de l'ennemi, connaître l'avenir... (17). Mais elle n'est pas seulement un outil à la disposition des chamanes, elle touche à tous les aspects de la vie des peuples qui l'utilisent : elle est utilisée traditionnellement pour accéder à un état de conscience que les indigènes croient favorables pour effectuer un voyage dans le monde des esprits ou des morts (3). Les vision qu'elle engendre, sont mises (pour les utilisateurs) sur le compte d'une capacité paranormale de relation avec «l'autre monde» (des ancêtres, des dieux...), ou de communication à distance.

L'absorption de 2 à $3 \mathrm{dL}$ de cette préparation provoque après 20 à 30 minutes environ, l'apparition de visions colorées comparables à celles obtenues avec la mescaline, la psilocybine ou le LSD (mais l'image du corps n'apparait pas perturbée, à la différence des symptômes provoqués par le LSD et la mescaline). La durée d'action peut varier de 2 à 6 heures selon la nature du mélange de plantes (17).

Les effets de l'ayahuasca ont été étudiés entre autres par Riba et coll., publiés en 2003 (18), ces auteurs ont plus précisément évalué les effets cardiovasculaires et l'excrétion urinaire de métabolites de la monoamine. L'étude menée sur 18 volontaires ayant déjà eu une expérience de consommation d'ayahuasca a montré que les effets maximum étaient ressentis entre 1,5 et 2 heures après la prise et la concentration sérique maximum de DMT était mesurée 1,5 heure après la prise. Les modifications observées concernaient la perception, des variations de l'humeur, une augmentation significative de la pression artérielle diastolique aux fortes doses, alors que la pression systolique et le rythme cardiaque étaient modérément ou peu significativement augmentés. 
Que l'ayahuasca contienne uniquement B. caapi ou également des additifs végétaux, il est nettement purgatif, psychoactif et psychédélique. Les effets communément décrit par les consommateurs d'ayahuasca sont d'ordre digestif voire purgatifs (nausées, vomissements parfois violents, diarrhées), on observe également vertiges, transpiration, états soit euphorique, soit agressif, magnification de la perception des sons, des couleurs, des contours (détails), sensibilité exacerbée aux odeurs, perméabilité à l'Autre (effets observés entre autres par F. Bois-Mariage (2)), hallucinations visuelles qui diffèrent selon les additifs végétaux choisis avec fréquemment des visions notamment d'animaux. Puis une période de sommeil fiévreux peut s'ensuivre. La présence de tryptamines est susceptible d'engendrer des tremblements convulsifs, une mydriase, une tachycardie. Après consommation d'ayahuasca, on observe des élévations de la fréquence cardiaque et de la pression artérielle durant les 60 à 90 minutes suivant la prise, puis un retour à la normale, voire une légère bradycardie et hypotension. Ces élévations de fréquence cardiaque et de pression artérielle sont deux fois moins importantes (19) qu'après une administration de DMT de synthèse. Cette modération des effets cardiovasculaires est volontiers attribuée à l'effet hypotenseur et bradycardique de l'harmine connu depuis longtemps (20).

\section{Virola}

Contrairement aux Indiens de Colombie qui la réserve au Chamanes, certaines tribus du haut Orénoque vénézuélien et brésiliennes consomment la drogue extraite de cette plante encore appelée Epena, Ebena, Nyakwana... très couramment. Tous les hommes au dessus de treize ou quatorze ans y sont autorisés occasionnellement. Au cours d'au moins une cérémonie annuelle, ils prisent la poudre obtenue après extraction à partir de l'écorce, sans discontinuer pendant deux ou trois jours de suite. Les principes hallucinogènes sont surtout présents dans le liquide exsudé par la surface intérieure de l'écorce (tendres couches du cambium) lorsqu'elle est retirée de l'arbre. L'analogie structurelle et chimique des principes actifs de Virola permet de comprendre la similitude des effets cliniques observés après consommation de Virola et d'ayahuasca.

L'effet de cette drogue est également très marqué : agitation et excitabilité intenses suivies d'une dépression voire d'une perte de conscience (1).

\section{Toxicité de l'ayahuasca}

Il est important de noter qu'aucun cas de décès suite à l'ingestion d'ayahuasca n'a été rapporté dans la littérature scientifique consultée.

L'une des seules complications sérieuses documentées est l'interaction entre l'ayahuasca et les antidépresseurs
Inhibiteurs Sélectifs de la Recapture de Sérotonine (ISRS) tels que la fluoxétine (21), cette observation est majeure car cette classe de psychotrope est largement prescrite dans le monde. Callaway et Grob (20) mettent en garde contre une interaction pouvant entraîner le décès suite à un syndrome sérotoninergique grave. Il apparaît donc nécessaire de rappeler qu'avant une ingestion d'ayahuasca il faut impérativement procéder à l'arrêt des prises de tout médicament augmentant le taux de sérotonine cérébrale libre (inhibiteurs sélectifs ou non de la recapture de sérotonine, IMAO-A ou non spécifique, précurseurs de la sérotonine).

En ce qui concerne la toxicité à moyen ou long terme d'une consommation d'ayahuasca, une étude a été menée par une grande équipe internationale (chercheurs brésiliens, américains et finlandais) et a conclu que les 15 membres d'un culte syncrétique brésilien (l'União do Vegetal, UDV) observés, ne présentaient pas d'anomalies physiologiques, neurophysiologiques ou psychiatriques alors même qu'ils avaient consommé de l'ayahuasca environ une fois par semaine durant plus de dix ans à l'occasion de cérémonies du culte. L'équipe de chercheurs a tout de même mis en évidence chez les personnes testées par rapport aux sujets du groupe de contrôle, une augmentation significative du nombre de sites de recapture de la sérotonine au niveau des plaquettes sanguines (22).

En somme, il n'existe à ce jour pas de toxicité aiguë décrite concernant l'ayahuasca ; toutefois des crises psychopathologiques ont parfois été décrites. Ces crises d'allure psychotique, de type bouffée délirante subaiguë, ont été observées plusieurs fois, tant chez des consommateurs occasionnels que chez des praticiens expérimentés de l'ayahuasca (faits plus rare). En France, F. Bois-Mariage rapporte deux épisodes délirants survenus l'un après l'autre au cours d'une session d'ayahuasca (2). La prévalence de ce type de crises reste faible mais mérite d'être signalée d'autant que celles-ci auraient plus de risques d'apparaître chez des consommateurs naïfs que chez les habitués et lorsque la préparation à la session est courte (1 à 2 jours).

\section{Observation médico-légale}

La littérature scientifique consultée à ce jour n'en a mentionné aucune.

\section{Utilisation contemporaine}

Depuis peu, un fort engouement pour l'ayahuasca est né dans nos sociétés occidentales, phénomène relayé par un accès facile sur Internet. Des «séminaires d'évolution personnelle» sont nés s'inscrivant dans un désir de quête spirituelle, et semblent obtenir un franc succès. Pour preuve, des voyages d'initiation à l'ayahuasca d'environ une semaine vers l'Amérique du Sud (Brésil, Pérou, Colombie) sont organisés et proposés 
sur Internet. De plus il est relativement aisé de se procurer l'ayahuasca, (ou les plantes constituant cette boisson), en vente sur différents sites Internet à des tarifs autour de $20 \$$ US les 100 grammes de vin de l'esprit (23).

\section{Soumission chimique}

En dehors du cadre historique et protohistorique de la tradition indigène, des «églises» basées sur la consommation rituelle d'ayahuasca sont nées au Brésil dès 1930 avec l'afflux de colons dans le bassin de l'amazone. Ces dernières années, au sein de ces sectes dénommées «églises» par les adeptes, en Europe (Suisse, Allemagne, France, Belgique, Espagne) et aux EtatsUnis, plusieurs saisies, arrestations et mises en examen ont eu lieu pour avoir chimiquement soumis des adeptes et détenu des substances illicites. En effet au regard de la loi et selon les pays, certains des principes actifs précités sont classés comme stupéfiants (la DMT est une substance contrôlée aux USA depuis 1985). Le ministère de la Santé Publique français à classé, la DMT et ses dérivés comme produits stupéfiants. L'harmine et ses dérivés ainsi que l'ayahuasca sont en cours d'évaluation par la commission des stupéfiants de l'Agence Française de Sécurité Sanitaire des Produits de Santé (AFSSAPS).

\section{Conclusion}

Indépendamment des désordres psychologiques et psychiatriques que peut entraîner l'ayahuasca, la consommation de boisson de ce type, en dehors du cadre traditionnel, peut permettre des escroqueries et des manipulations ; en particulier le risque de soumission chimique organisée en groupe n'est pas négligeable face à des produits ayant un tel potentiel psychotrope et pouvant occasionner des délires psychotiques. Des dérives présentant un risque pour la santé, ont notamment pu être observées en Europe dans le cadre de sectes qui droguaient leurs adeptes à l'ayahuasca sans la préparation psychologique et physique préalable nécessaire pour en diminuer les effets toxiques somatiques à court et à moyen terme. Cette mise en conditions (jeûne préalable, repos compensateur ultérieur, etc....) est d'usage dans les sociétés primitives et indispensable afin que la consommation occasionnelle d'ayahuasca présente un minimum de risques.

\section{Remerciements}

Les auteurs tiennent à remercier vivement L. Rivier, consultant scientifique à Lausanne pour ses précieux renseignements et Frédéric Bois-Mariage, psychologue, co-doctorant à Paris VII, pour son excellente synthèse interdisciplinaire sur l'ayahuasca.

\section{Références}

1. Bruneton J. Alcaloïdes - Dérivés du tryptophane. Tryptamines, $\beta$-carbolines. In : Pharmacognosie, phytochimie, plantes médicinales. Paris : Editions Tec\&Doc, Editions Médicales Internationales, 1999 : 967-74.

2. Bois-Mariage F. Ayawaska : une synthèse interdisciplinaire. Psychotropes : Revue internationale des toxicomanies et des addictions. $2002 ; 8$ (1) : 79-113.

3. Schultes RE, Hofmann A. Les plantes des dieux. Paris : Lézard Ed., 1993 : 120-7.

4. Rivier L., Lindgren J.E. «Ayahuasca», the South American hallucinogenic drink: an ethnobotanical and chemical investigation. 1972 ; Economic Botany ; 26 : 101-29.

5. McKenna D.J., Towers G.H.N. On the comparative Ethnopharmacology of Malpighiaceous and Myristicaceous hallucinogens. J. Psychoactive Drugs. 1985; 17: 35-9.

6. Schultes R.E., Hofmann A. Plants of hallucinogenic use. In: The botany and chemistry of hallucinogens. Springfield, Il, USA: C.C. Thomas Publisher, 1979 : 12239.

7. Holmstedt B. et Lindgren J.E. Chemical constituents and pharmacology of South American Snuffs. In Efron D., Holmstedt B.,Kline N.S. (Eds) Ethonpharmacological Search for Psychoactive Drugs. Public Health Services $N^{\circ} 1645$, Washington DC US Gov. Printing Office, 1967 : 339-73.

8. Callaway J.C., Raymon L.P., Hearn W.L., McKenna D.J., Grob C.S., Brito G.S., Mash D.C. Quantitation of N,Ndimethyltryptamine and harmala alkaloids in human plasma after oral dosing with ayahuasca. J Anal Toxicol. 1996 ; 20 (6) : 492-7.

9. Yritia M, Riba J, Ortuno J and al. Determination of N,Ndimethyltryptamine and $\beta$-carboline alkaloids in human plasma following oral administration of Ayahuasca. J. Chromatogr. B. $2002 ; 779$ : 271-81.

10. Angrist B., Gershon S., Lopez-Ramos B., Mandel L.R., Sathananthan G., and al. Dimethyltryptamine levels in blood of schizophrenic patients and control subjects. Pharmacology. 1976 ; $47: 29-32$.

11. Wyatt R.J., Mandel L.R., Ahn H.S., Walker R.W., Vanden Heuvel W.J.A. Gas chromatographic-mass spectrometric isotope dilution determination of N,N-dimethyltryptamine concentrations in normals and psychiatric patients. Psychopharmacologia. 1973 ; 31 : 265-70.

12. Ciprian-Ollivier J., Cetkovitch-bakmas M.G. Altered consciousness states and endogenous psychoses : a common molecular pathway? Schizophrenia Res. 1997 ; 28 : 257-65.

13. Smythies J.R., Morin R.D., Brown G.B. Identification of diméthyltryptamine and O-methylbufotenine in human cerebrospinal fluid by combined gas chromatography/mass spectrometry. Biol. Psychiatry. 1979 ; 14 : 549-56.

14. Zheng W., Wang S., Barnes L.F., Guan Y., Louis E.D. Determination of harmine and harmine in human blood using reversed-phase high-performance liquid chromatography and fluorescence detection. Anal. Biochem. $2000 ; 279$ (2) : 125-9. 
15. Callaway J.C. carbolines endogenas y otros alcaloides indolicos en los mamiferos, in J.M. Feriegla (Ed.) Plantas, chamanismo $v$ estados de consciencia, Barcelona, Espagne, Los Libros de la Liebre de Marzo, 1994 ; 196-229.

16. Pépin G., Chèze M., Billault F., Gaillard Y. Un nouvel hallucinogène en Europe : l'ayahuasca ou vin de l'esprit. J Med Leg Droit Med. 2000 ; 43 (7-8) : 666-75.

17. Rivier L. Ethnotoxicologie de l'ayahuasca. Congrès CCCTA et SFTA, Martigny - Suisse. 19-22 juin 2002.

18. Riba J., Valle M., Urbano G, Yritia M, Morte A., Barbanoj M.J. Human pharmacology of ayahuasca: subjective and cardiovascular effects, monoamine metabolite excretion and pharmacokinetics. J Pharmacol Exp Ther. 2003 ; 306 (1) : 73-83.

19. Callaway J.C., McKenna D.J., Grob C.S., Brito G.S., Raymon L.P., Poland R.E., Andrade E.N., Andrade E.O., Mash D.C. Pharmacokinetics of Hoasca alkaloids in healthy humans. J. Ethnopharmacol.. 1999 ; 65 : 243-56.
20. Greeff K., Dènes B., Tawfik H. Cardiovascular effects of (+)- and (-)-tranylcypromine compared to other monoamine oxidase inhibitors. In: Beckmann H., Riederer P. (éd), Monoamine Oxidase and its Selective Inhibitors, Modern Problems of Pharmacopsychiatry, 19, Bâle : Karger. 1983 : 220-30.

21. Callaway J.C., Grob C.S. Ayahuasca preparations and serotonin reuptake inhibitors: a potential combination for severe adverse reactions. J. Psychoactive Drugs. 1998 ; 30 (4) : 367-9.

22. Grob C.S., McKenna D.J., Callaway J.C., Brito G.S., Neves E.S., Oberlander G., Saide O.L., Labigalini E., Tacla C., Miranda C.T., Strassman R.J., Boone K.B. Human psychopharmacology of Hoasca, a plant hallucinogen used in ritual context in Brazil. J. Nervous Mental Disease. 1996 ; $184(2): 86-94$.

23. www.bouncingb.com 